\title{
Integrating a learning management system with a student assignments digital repository: a case study
}

\author{
Francisco Javier Díaz*, \\ María Alejandra Schiavoni, \\ María Alejandra Osorio, \\ Ana Paola Amadeo and \\ María Emilia Charnelli
}

\author{
LINTI - New Information Technologies Research Laboratory, \\ Computer Science School, \\ National University of La Plata, \\ La Plata, Buenos Aires, Argentina \\ Email: jdiaz@unlp.edu.ar \\ Email: ales@info.unlp.edu.ar \\ Email: aosorio@cespi.unlp.edu.ar \\ Email: pamadeo@info.unlp.edu.ar \\ Email: mcharnelli@linti.unlp.edu.ar \\ *Corresponding author
}

\begin{abstract}
The integration of different platforms in the academic environment is a challenge within the field of information technology. In the field of e-learning, where learning management systems are used for courses development, their integration with other platforms and applications is interesting, in particular with digital repositories. This article describes the integration of Moodle with the DSpace repository. The integration process consists of two stages, in order to establish full communication to and from the repository. For communication from Moodle to the repository, with the goal of consulting and transferring elements from DSpace, modules already implemented were used. The current stage is implementing a specific module in order to establish communication in the other direction and allow transferring resources from the LMS to the repository. This module is very useful for educational material that can be made publicly available through a repository and thus transcending the borders of an educational environment.
\end{abstract}

Keywords: e-learning; learning management system; LMS; repositories; learning objects; university; Argentine.

Reference to this paper should be made as follows: Díaz, F.J., Schiavoni, M.A., Osorio, M.A., Amadeo, A.P. and Charnelli, M.E. (2015) 'Integrating a learning management system with a student assignments digital repository: a case study', Int. J. Continuing Engineering Education and Life-Long Learning, Vol. 25, No. 2, pp.138-150.

Biographical notes: Francisco Javier Díaz holds a BS in Applied Mathematics and a degree in Computer Estimation (Calculista Científico) from the UNLP. $\mathrm{He}$ has been an unsupervised Deputy Researcher with the Comision de Investigaciones Cientificas of the Province of Buenos Aires (CIC-BA) since 1990. In 1999, he became the first elected dean of the Computer Science School of the UNLP, and was re-elected in 2004. He is currently the General, 
Scientific and Technological Director of the Center for Information Processing (CeSPI) of the UNLP. He is an Associate Professor at the Computer Science School of the UNLP and also the Director of the Laboratory of Investigation into New Information Technologies (LINTI) which hosts 50 researchers and is the base of ten subjects of the BS in Computer Science and the MS in Data Networks, which he also directs.

María Alejandra Schiavoni holds a BS in Computer Science and a degree in Computer Estimation (Calculista Científico) from the UNLP. She has been carrying out duties as a teacher and a researcher since 1987. She is currently an Associate Professor of the Computer Science School of the UNLP. Since 1992, she has been a member of the Laboratory of Investigation into New Information Technologies (LINTI) of the Computer Science School, where she conducts research. Since 2008, she coordinates and prepares students for ACM ICPC programming competitions. Since 2010, she serves as the Communications and Promotions Director of the Computer Science School. Her articles have been published in prestigious national and international scientific events of the field.

María Alejandra Osorio holds a BS in Computer Science from the UCA (Universidad Católica Argentina). She is currently a student of the MS in Virtual Learning Environments in Virtual Educa and University of Panama. As a member of the Laboratory of Investigation into New Information Technologies (LINTI), she coordinates the virtual platform that hosts the degree, postgraduate and extension courses. Her work has been published in prestigious scientific events and journals, both national and international. She currently serves as an Associate Professor of the Computer Science School of the UNLP and as the Director of Academic Systems at the CeSPI.

Ana Paola Amadeo holds a BS in Computer Science from the UNLP. She also holds the degree of Professor of the UNLP (2007). She is currently a student of the MS in Virtual Learning Environments in Virtual Educa and University of Panama. As a member of the Laboratory of Investigation into New Information Technologies (LINTI), she coordinates the virtual platform that hosts the degree, postgraduate and extension courses. Her work has been published in prestigious scientific events and journals, both national and international. She currently serves as an Associate Professor of the Computer Science School of the UNLP and as the Coordinator of Academic Systems at the CeSPI.

María Emilia Charnelli is an advanced student of the BS in Computer Science of the UNLP, and a Student Assistant at the same School. Since 2011, she has been an intern at the Laboratory of Investigation into New Information Technologies (LINTI), and is currently an intern at the Comisión de Investigaciones Científicas (CIC) of the Province of Buenos Aires.

This paper is a revised and expanded version of a paper entitled 'Integrating a learning management system with a student assignments digital repository. A case study' presented at the IADIS 2013, IADIS Multi Conference, Computer Science and Information Systems, Prague, Czech Republic, 23-26 July 2013. 


\section{Introduction}

In current times, information systems integration is paramount, and constitutes a challenge in the field of information technology. Systems are generally not designed for integration. Instead, they are typically developed to solve specific problems. Thus, each system uses languages and technologies of its own, according to the needs of each application, which makes communication between them difficult. Integration comes from the need to share data among heterogeneous systems, in order to achieve unification in information access and create the impression in users that they are interacting with a single system. An integral view allows for the retrieval and reuse of information through one single point of access.

There is a wide set of applications in multiple environments that benefit from information integration, for example, in the area of e-commerce and e-business, business services and transactions can be made simple through networks (Ziegler and Dittrich, 2004). In the field of e-learning, where learning management systems (LMSs) are used for course development, their integration with other platforms and applications can help increase resource availability and communication among users. In this field, learning objects (LOs) are the basis of interoperability, which is why integration among intervening systems is particularly important in this context. The possibility of integrating LMSs with general purpose digital repositories and learning object repositories (LORs) proposes a change in how teachers think, plan and build educational content (Pereira Rodrigues et al., 2011).

The Moodle LMS has been used in the school for over nine years. There is a great amount of stored academic material and a high number of users. Currently, in light of the new trends in educational material and with the growth of open educational resources (OERs), there arose the initiative of storing content in one single public space available for everyone. For this reason, DSpace began to be used as a repository to store LOs and all the content produced by the school, in order to facilitate its retrieval and reuse.

Likewise, the amount of users on Moodle has promoted initiatives for integrating the LMS with social networks extending the functionality of the platform so as to improve communication with the students through their social habits. Thus, all activity engaged in through the platform, including updates of content stored in it can be automatically notified through the multiple social networks used in the school (Díaz et al., 2012a). The LMS also integrates with the academic management system SIU-Guaraní of the Computer Science School with the goal of sharing courses and users. By syncing the management system and the educational tool, the students from different subjects automatically integrate the course within the Moodle platform (Díaz et al., 2012b).

This article describes the integration of the Moodle LMS with the DSpace repository, both from and to the repository. It will describe in detail the implementation of the module that will allow communication towards DSpace for the transference of LOs from the LMS to the repository. The transference from DSpace to Moodle was solved through the Rest API provided by DSpace and a specific module available in Moodle (Ochoa Agüero et al., 2010). 
Figure 1 Platforms and systems that are integrated with Moodle

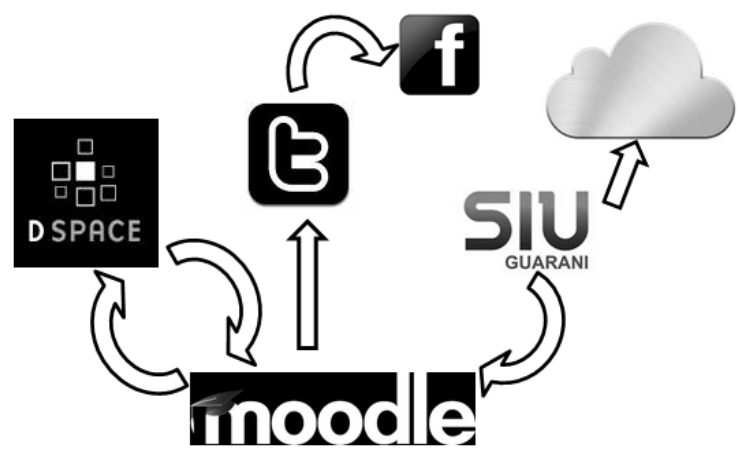

The first stage consists of the development of the module that allows communication from Moodle to DSpace for a particular use case that is the publication of work done by the students and stored in the LMS through the tasks functionality in Moodle. This paper will describe the way in which the resource is prepared for its publication and the process of incorporation of the metadata. In order to show the work context, we will also describe the use of the LMS in the school as a fundamental e-learning tool for the dissemination of educational material and the advantages of the use of a repository for storing the resources generated within the institution.

\section{OER initiative}

The term OER was coined in the 2002 UNESCO forum on the incidence of Open Courseware and describes teaching, learning and research materials in any storage medium, digital or otherwise, which are in the public domain or have been published with an open license that allows free access to these materials, as to their use, adaptation and redistribution without restrictions or with limited restrictions (COL, 2010).

The goal is to support the transformation that is taking place in education from lifelong learning, the needs and interest of the students, the flexibility and the facility with which these digital objects are shared through the internet with an appropriate license (Butcher, 2011). The fundamental role of universities is to generate and use OERs developed by third parties. Adapting existing OERs to local cultural and learning characteristics, thus generating new OERs with similar licenses allows for a significant reduction in the costs implied by the development of new educational content, while improving the quality of the curricula by including material generated by prestigious universities from around the world (COL, 2010). UNESCO has taken on a fundamental role in this initiative, promoting the debate around its practical application with the goal of encouraging the access, equity and equality promoted in the Universal Declaration of Human Rights.

There are several OER repository initiatives in the world involving governmental proposals, such as teacher education OER Africa, academic such as MIT OpenCourseware, which provides material and full subjects given at MIT; the Merlot repository, Multimedia Educational Resource for Learning and Online Teaching http://merlot.org, OER Commons, http://www.oercommons.org/ with over 47,000 open resources and curatorship and training services, and Europe's Learning Resource 
Exchange for Schools, with over 50 organisations generating creative commons licensed content, just to name a few.

The Computer Science School of the UNLP has adhered to this initiative in two ways. First, by generating LOs out of material created by the teachers of multiple subjects, as is the case with the web accessibility course offered each year since 2011 as a distance learning subject and which has raised great interest from multiple actors of society (Díaz et al., 2012c). The second line has been the construction of OERs from the material generated by the students, which is the subject of this paper.

\section{DSpace digital repository}

The current academic and scientific development of all universities increases the general production of scientific material and consequently generates a need to group, store, preserve and distribute great amounts of information in a timely manner. Thus, it gives rise to new ways to store information, such as the digital library at first, and later, the digital repository, a more global and abstract method. Digital repositories are virtual spaces that store sets of documents in multiple digital formats, organised according to pre-established criteria (Ochoa Agüero et al., 2010).

There are currently a number of initiatives that develop and encourage the use of repositories in general and LORs in particular, a fact that can be verified by checking the repositories that are available online, with tens of thousands of resources in storage. There are also initiatives that are working towards interoperability between repositories, with the goal of generating networks of distributed systems for general searches (Díaz et al., 2011a).

In our case, the institutional repository was created out of a need to store in a single publicly accessible location the material produced by the school that deserves global distribution. Consequently, a repository was implemented through DSpace, an open source application that offers a wide range of functionality for resource management. The first stage will consist of the storage of projects developed by students for one of the subjects of the school. These projects are sometimes assigned by the teachers, or written by students who become especially interested in a certain topic and choose to develop a related application.

If the subject contemplates this option, the best projects can be used with real users to meet specific demands of educational or health institutions or non-profit organisations, such as, for example, a system that kept the statistics of a local soccer team, or one that kept a record of the resources in the historical archive of the Province of Buenos Aires, to name a few examples. Later stages contemplate the incorporation of LOs designed for courses, final reports, and degree theses.

In order to store the aforementioned educational material, a structure of collections will be created and divided into subjects. The resources will be classified using descriptive metadata related to the characteristics of the project, such as topic, platform used, installation requirements and use cases, among others determined by the professor and by other actors such as librarians and students. The metadata can be updated after the publication of the resource, with the goal of improving searches and granting permanent validity to the repository.

The possibility of permanently and publicly storing these projects will allow other students to become acquainted with them and will serve as a basis for their further 
development. These developments can be also used by the teachers of other subjects aiding as examples of applications and technologies in use. The repository that was implemented at the school, DSpace version 1.8, is currently beta in design and content, and can be found at http://dspace.linti.unlp.edu.ar.

\section{Moodle virtual platform}

The school has been working with the Moodle virtual platform for online course management for over seven years as a complement to in-person undergraduate and postgraduate classes, as well as courses offered by the Secretary of Extension. The platform includes over 10,000 registered users in around 170 courses.

The study material is comprised of digital resources in multiple formats. For example, some extension courses have experimented with the use of standardised virtual classes through LOs. The possibility of using LOs that comply with the SCORM standard allows not only reusing them, but also following and keeping a record of the progress of each student. All the content is also published in HTML, PDF and slideshow format. Some cases include videos that work as triggers for new topics or as complements to an explanation. These videos are published in the platform and linked through the resource of a website. Software is also included when necessary in the proposed activities, as well as links with extra contents and ad-hoc specific material for web developers (Díaz et al., 2011a, 2011b). Figure 2 shows the used of multiple resources in the platform through the years.

Figure 2 Resources used in the Moodle virtual platform

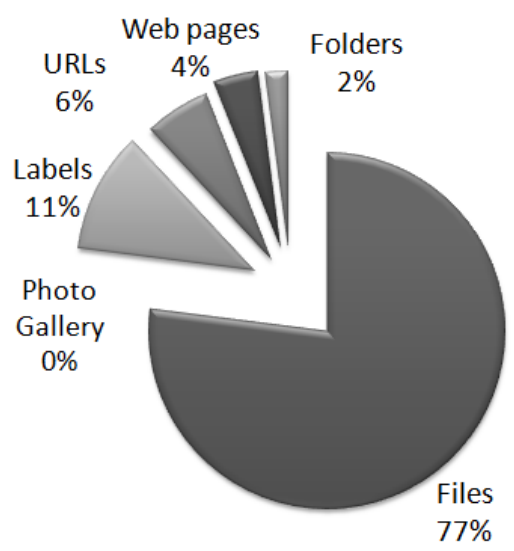

Content distribution, both theoretical and practical, is achieved mostly through files in multiple formats such as PDFs, text, and slides, among others. These files, together with tags for course organisation and URLs for further information, make up the three most used types of resource in the virtual platform.

Activities include discussion forums, used for communication between students and teachers, and in some cases for general notifications, such as exam dates or other important information. Questionnaires have been used in many subjects for approximately two years, and are very useful for self evaluations or discussions of 
specific topics, in mass courses. Graduate courses tend to use them before virtual encounters in order to level contents so as to better seize them. Choices are used, for example, to survey the general opinion of the students on an activity or a class or specific administrative matters, or even to measure attendance to a mid-term. Figure 3 shows the use of the activities in the courses. As seen, discussion forums are the most used, mainly for communication with the students, both in academic and administrative matters. Assignments come second and are the central motivating axis of this paper. This workgroup has also developed a social networking connection module, a Twitter Activity Module, growing in use for communication between students and teachers.

Figure 3 Activities used in the Moodle virtual platform

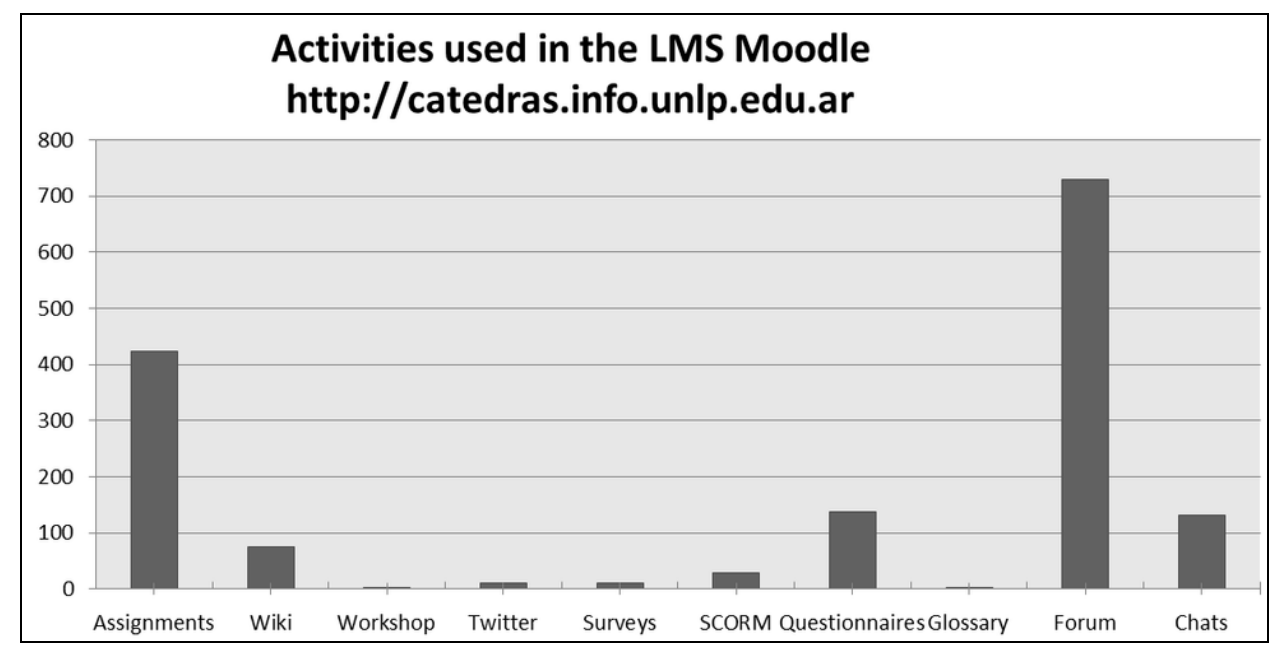

Assignment is one of the most used modules because of the practical nature of the courses that habitually use the virtual platform. Through assignments, students can upload files that can later be evaluated by their teachers, who grade and give feedback through Moodle. Grade and feedback are sent to students by e-mail and can only be seen by them and the teachers. This is useful both for teachers and students, as the latter can upload their work online from home and any time before the established deadline.

Depending on the subject, the projects developed by the students have different magnitudes. In order to correct them, the teachers must have the correct environments in order to install or unzip and execute them for testing and error detection. In the case of subjects from early years such as the language seminar, where either $\mathrm{C}$ or Python is taught, the projects are simple, in some cases consisting only of programmes that solve a particular problem. In these cases, the correction process is relatively simple, as a complex environment is not required for their execution.

In later subjects such as software laboratory or software project, the projects are bigger and generally consist of integral systems involving more sophisticated developments with access to data bases. In these cases, following and correcting the projects is more difficult for the teachers as they involve some difficulties related to the replication of the environment the students use for development. For this reason, all the subjects that require it have had access for five years to a server where students have all the products installed and configured to develop their projects. For example, software 
project has an SVN or subversion server for digital document version control. It maintains a record of all the work and changes made to the source code files conforming a project. The SVN server also holds a MySQL database server, accessible through PHPMyAdmin, which has the data bases necessary for the projects already defined. The delivery of the tasks is in groups, so each group accesses the SVN server through a pre-established user. For the development of the project, the students access the user view of their system through a specific web address which includes their group number. This same space is used by the teachers when they correct the work (Diaz et al., 2008).

This work methodology is very useful both for the students and for the teachers, and it is used in other subjects as well. For publishing these kinds of projects that involve multiple technologies aside from source codes and a data base model, it is mandatory to include additional information about the products used, their versions and installation guides. In cases where the projects are even more complex, the material sent through the LMS to the repository requires a set of guidelines that allow full and consistent publishing of the project, which is out of the scope of this paper.

As previously mentioned, some projects are developed with a specific goal in mind and for use in one institution that requires them. This makes the resulting systems of interest beyond the subject that generates them, where only specific knowledge is evaluated. They are also sometimes used as material for the course in the years that follow, although these are rare cases and generally discarded each year. Taking the amount of total projects uploaded to the platform into account, which is over 2700 , the waste is significant and there is high reuse and re-signification potential within the same course or by other educational institutions that find it useful.

\section{Communication between DSpace and Moodle through the sword protocol}

As mentioned in previous sections, both the Moodle LMS and the DSpace repository use their own technologies according to their needs, and in many cases their integration is not immediate, despite the increasing flexibilities they have been offering through time.

The development of the Moodle platform has been continuously growing. Moodle Version 2.0 incorporated the concept of repository and communication with other well known repositories such as Alfresco, Merlot, Flicker, Google Drive, Picasa, and Dropbox was implemented. This feature considerably increased flexibility in file handing and access to external repositories within the platform itself. Although this extension is very useful, it is important to highlight that communication is always established in one direction only, from Moodle.

The initial goal was to obtain communication from Moodle to the repository, with the goal of retrieving and transferring elements from DSpace. Modules were used to extend the functionality of the platforms that would be involved in the new communication channel. In the repository, a module that installs an API using REST and that is available through the DSpace community was used. The LMS has a Repository API that was used to incorporate a specific plugin to DSpace, created by the Moodle community.

In relation to the other communication channel, a new functionality was implemented that makes it possible to publish content from the Moodle platform to different repositories. This new functionality is very useful to export all kinds of material from the 
courses, such as theoretical or practical content, documentation, and projects delivered by the students. It is also possible to export standardised content as LOs.

The development of this tool involved the Sword protocol, which implements simple remote storage of resources into a repository from other applications.

In our application case, the functionality was incorporated from the interface of the Assignment module in Moodle, which allows for automatic delivery of these assignments. Figure 4 shows the send to repository link (Enviar al repositorio) on the upper left corner, circled in green. This link will establish communication between the LMS and the repository, described in detail in later sections.

Figure 4 Interface for the visualisation of Moodle tasks, with the send to repository link

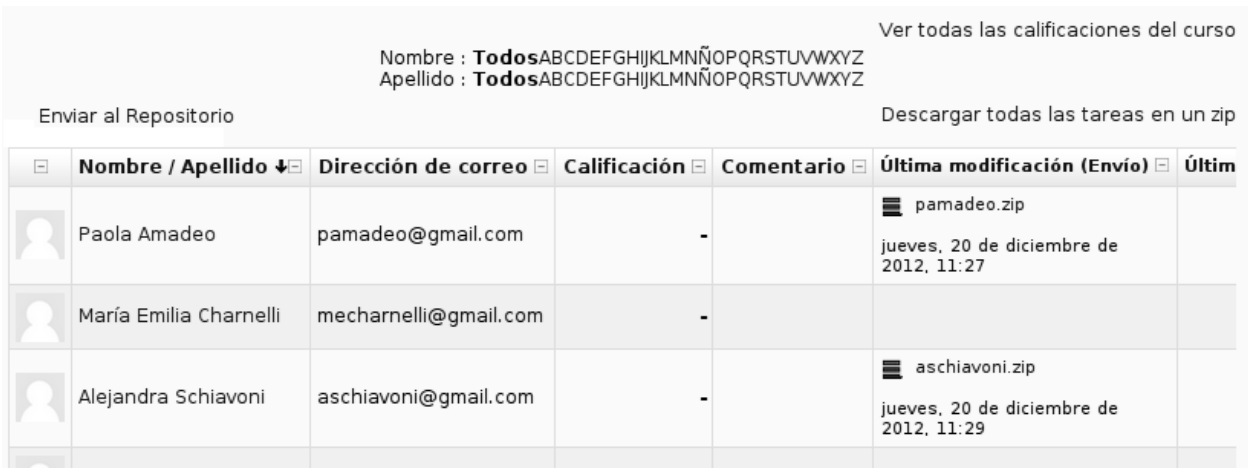

\subsection{Metadata assignment}

The incorporation of resources into a repository requires the generation and instantiation of metadata that act as indices to locate technological resources. Making use of the right metadata guarantees fast and effective tracking of a sought resource and enables greater production visibility. In the case of the presented repository, cataloguing the resources generated by the students is done through a specific module and uses its own metadata format, given that Moodle does not implement its own metadata format.

The information for each course, resource and assignments is stored by Moodle in the database and can be used for generating metadata in any established standard, in our case Dublin Core, the standard used by DSpace. Afterwards, depending on the metadata defined for sending to the repository, the information is retrieved and is sent together with the resource to DSpace. In the case of the works by the students, the context information of the Task is taken as metadata, including course name, year, teacher in charge, teacher e-mail address and data about the task itself such as author or authors, e-mail addresses, grade, comments by the teachers and assignment. Implementing text mining can result in the retrieval of specific information and key words that allow for a more detailed classification of the material to aid future queries. Figure 5 shows the cataloguing process and its actors. 
Figure 5 Resource cataloguing shared among authors (students), revisers (teachers) and additional revisers (librarians)

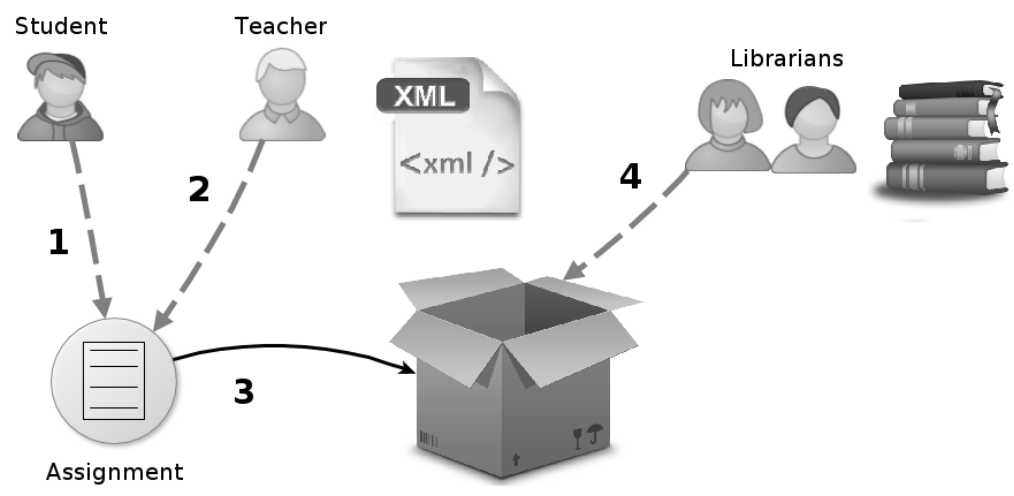

It is important to emphasise that while most of the data is extracted automatically, the teacher and the student can incorporate additional information such as additional authors, platform and programming languages used for coding the resource.

The repository can also be accessed through Meran, a free software system developed by the UNLP for full library user and resource management. Librarians can also catalogue these resources as part of a certain specialty, by means of virtual shelves associated with a course, numbering, among others.

\subsection{The SWORD protocol}

The simple web-service offering repository deposit (SWORD) protocol is an atom publishing protocol (APP) application that defines simple remote content storage into a repository from other applications.

With only two basic operations, SWORD creates services that offer functionalities such as deposit from multiple locations or from standard applications, and multiple storage to different repositories. The availability of SWORD libraries in multiple languages, such as PHP, Java, Python and Ruby promotes the use of this kind of integration. DSpace, Fedora Eprints, IntraLibrary, and DataBank are examples of repositories that implement this service.

An interesting aspect of this protocol is that the sending service can be configured so that any user can upload information directly to the repository without logging in. This solves the problem of worrying about the amount of users or permissions, as this process would be managed through the application, and later, a qualified repository user could validate and complement the received data.

\subsection{Implementing communication}

After analysing the architecture of DSpace it became apparent that no implementation or installation was necessary, as the repository incorporated the communication functionality through this protocol. In version 1.8, DSpace implemented the protocol in two ways, as a server and as a client, both compatible with SWORD v2. 
In contrast, Moodle does not offer this communication functionality, which is why it was necessary to use the client API provided by SWORD to implement a specific module that will retrieve the requested information and prepare it according to the standards established by the protocol, to send it to the repository.

For our application case, the implemented module must retrieve the file stored in the Moodle assignment and then build the package in the SWORD format with the corresponding metadata. Following, we describe the process of package creation, metadata assignment and package sending between the two platforms.

\subsubsection{Creating the package}

As previously mentioned, Moodle does not have a metadata standard, it stores basic information in its database tables, which can be used to generate metadata in an established standard such as Dublin Core in our case.

Moodle has its own classes to handle the file system and the files themselves. When the requested file or files are retrieved through the stored file class, resource data is retrieved including name, creation date, last date of modification, author, format, and type of resource within Moodle, among others. Information such as assignment name, course name, and platform name can be extracted from resource context data.

The application generates a metadata encoding and transmission standard (METS) package with files and administrative, descriptive and structural metadata that the repository will use to incorporate the resource. The metadata are placed in an xml file that includes: a heading, descriptive metadata in MODS format, Creative Commons license, list of files and structural map. Later, this set of files will be packed in a single zip file.

\subsubsection{Sending the package}

The package is sent through the instantiation of a client of the PHP API provided by SWORD. The function deposit is used to automatically deliver it to the repository. It is necessary to specify in this function the URL of the repository, an access account and password if necessary, the content format, the destination collection and the zip file with the content. Figure 6 shows the steps followed when sending a resource from Moodle to DSpace.

Figure 6 Communication scheme between Moodle and DSpace through SWORD

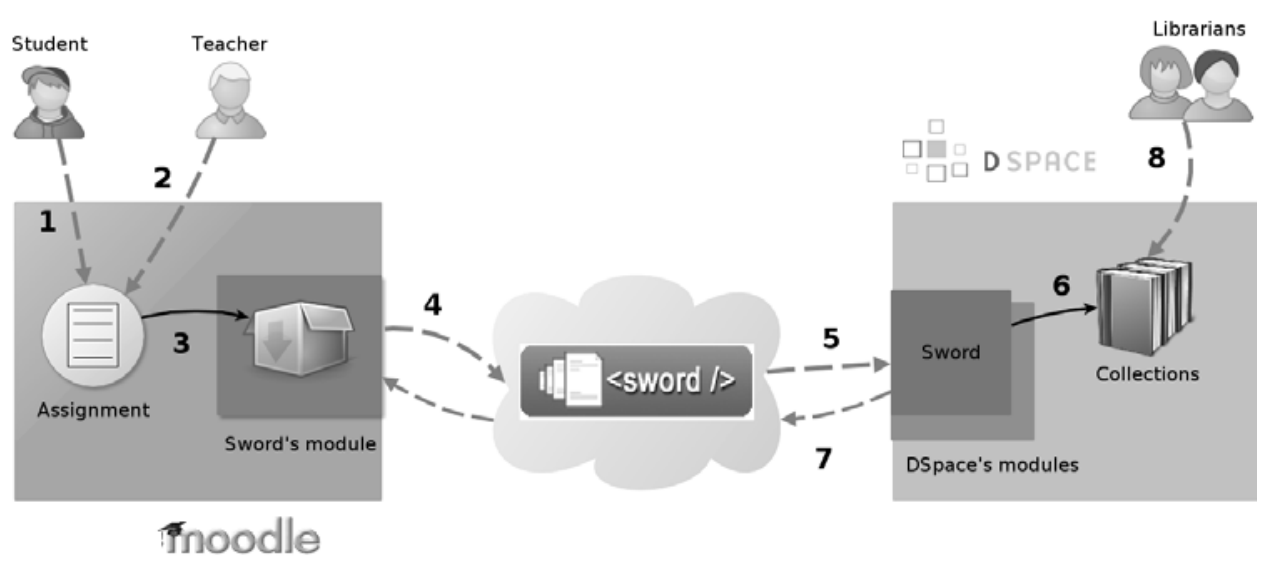


The steps are as follows:

1 The students deliver their assignment by uploading a file.

2 The teacher follows the deliveries and the corresponding corrections, sending feedback to the students when necessary. Once the delivery is finished, the teacher can choose to send it to the repository.

3 Once the teacher decides to make a delivery, the module comes into play. It takes each one of the assignments and packs them with their corresponding metadata into a zip file.

4 The package is sent through SWORD to a specific collection in the chosen repository.

5 The default SWORD module in DSpace unpacks it and retrieves the Dublin Core metadata and files.

6 Each of the files is added as items to the specific collection.

7 DSpace's SWORD module sends an xml response to the Moodle newly created module indicating operation status.

8 Librarians catalogue the resources.

After sending, the complete resource is stored in the corresponding collection within the repository, with its associated metadata. The result of this operation is the same as if the item was added manually following the steps required by DSpace for resource storage.

\section{Conclusions}

In e-learning, communication among platforms facilitates exchange, integration and reuse of educational resources that have been developed in heterogeneous platforms and tools. Standard creation and adoption established the use of certain norms for digital content creation and communication among systems. The growth of distance education has resulted in users placing emphasis in technical aspects related to connecting and using distributed resources in other platforms such as digital repositories.

The communication implemented between the Moodle LMS and the DSpace repository allows for a reduction in the overload of storing the same resources in multiple platforms. This encourages the teachers that manage their courses in the educational platform to publish one or more student projects delivered in one or multiple external repositories, as they do not need to be familiar with their interface and mode of access. This functionality encourages dynamic and automatic OER generation and potential reuse. Moreover, the module implemented prepares the information to be transferred by incorporating metadata with course context information, in the standard format used by destination repositories. Semi-automatic publishing of material is more practical as it is not necessary to follow all the steps required within the repository to upload the resource and complete the metadata that are indispensable for search and retrieval. In our case, the module is being tested with the DSpace repository, and the transferred resource remains with its basic Dublin Core metadata complete. The functionality of this module can be extended to other repositories, adapting the way in which material is organised, taking 
into account the standards used in each specific platform. To incorporate specific metadata that match other criteria and goals classification is planned the intervention of an interdisciplinary group of librarians who contribute their expertise on cataloguing. In our project, librarians participation is done at a later stage in the teacher publishing process. This will complete the registration of metadata with resource specific information from external aspects and offer different contribution when performing searches.

\section{References}

Butcher, N. (2011) A Basic Guide to Open Educational Resources (OER) COL, September 2011, UNESCO, ISBN: 9781894975414.

COL (2010) Taking OER Beyond the OER Community [online] http://www.unesco.org/new/en/ unesco/events/education-events/?tx_browser_pi1[showUid] $=2761 \& \mathrm{cHash}=92 \mathrm{cecc} 21 \mathrm{f8}$ (accessed 2013-08-01).

Diaz, J. et al. (2008) Utilizando herramientas de software libre para la gestión de cursos de grado, TE\&ET Revista Iberoamericana de Tecnología en Educación y Educación en Tecnología, Facultad de Informática, UNLP, pp.62-67, ISSN 1850-995.

Díaz, J. et al. (2011a) 'Conformando un Repositorio Digital de Acceso Abierto a partir del material académico', Proceedings of WICC 2011 - XIII Workshop de Investigadores en Ciencias de la Computación, Rosario, Argentina, pp.932-936.

Díaz, J. et al. (2011b) 'Desarrollo de Objetos de Aprendizaje sobre Accesibilidad Web: un caso de estudio comparando dos Metodologías de Diseño', Proceedings of the 2nd International Symposium on Innovation and Technology, ISIT2011, 1st ed., Lima, Perú, pp.60-65.

Díaz, J. et al. (2012a) 'Integración de Moodle con las redes sociales', Presentación del módulo Twitter Activity Module y su uso dentro de la plataforma, MoodleMoot UY 2012, Montevideo, Uruguay.

Díaz, J. et al. (2012b) 'Integración de Plataformas Virtuales de Aprendizaje, Redes Sociales y Sistemas Académicos Basados en Software Libre', Una Experiencia en la Facultad de Informática de la UNLP. Proceedings of SSI 2012 Simposio sobre la Sociedad de la Información, JAIIO, La Plata, Argentina, pp.58-70.

Díaz, J. et al. (2012c) 'Web accessibility learning objects development and evaluation: a case study', Accepted for publication in Proceedings of Sloan Consortium 5th Annual International Symposium for Emerging Technologies for Online Learning, Las Vegas, Nevada, EE.UU.

Ochoa Agüero, A. et al. (2010) 'Repositorios de Objetos de Aprendizaje de Acceso Abierto para la Educación de Postgrado', Revista Cubana de Informática Médica, República Bolivariana de Venezuela.

Pereira Rodrigues, A. et al. (2011) 'The use of web services as a strategy for integration of LORs to LMSs', Proceedings of XVII Congreso Argentino de Ciencias de la Computación, CACIC 2011, La Plata, Argentina, pp.1234-1243.

Ziegler, P. and Dittrich, K.R. (2004) 'Three decades of data integration - all problems solved?', 18th IFIP World Computer Congress (WCC 2004), Building the Information Society, Toulouse, France, Vol. 12, pp.3-12. 